\title{
Acupuncture for radiation-induced toxicity in head and neck squamous cell carcinoma: a systematic review based on PICO criteria
}

\author{
Pierluigi Bonomo ${ }^{1}$ (D) Giulia Stocchi ${ }^{1}$ - Saverio Caini ${ }^{2} \cdot$ Isacco Desideri $^{1} \cdot$ Veronica Santarlasci $^{3} \cdot$ Carlotta Becherini $^{1}$. \\ Vittorio Limatola ${ }^{3}$. Luca Giovanni Locatello ${ }^{4}$. Giuditta Mannelli ${ }^{5}$. Giuseppe Spinelli ${ }^{6}$. Carmelo Guido ${ }^{7}$. \\ Lorenzo Livi $^{1}$
}

Received: 18 June 2021 / Accepted: 14 July 2021 / Published online: 31 July 2021

(c) The Author(s) 2021

\begin{abstract}
Purpose In head and neck squamous cell carcinoma (HNSCC), the potential mitigating effect of complementary medicine interventions such as acupuncture for radiation-induced toxicity is unknown. This study aimed to assess the impact of acupuncture on the incidence and degree of severity of common radiation-induced side effects.

Methods In accordance with pre-specified PICO criteria, a systematic review was performed. Two electronic databases (Medline and Embase) were searched over a 10-year time frame (01/01/10 to 30/09/20). Patients undergoing a curatively intended, radiation-based treatment for histologically confirmed squamous cell carcinoma of the nasopharynx, oropharynx, larynx, hypopharynx and oral cavity represented the target population of our study. Accurate information on the acupuncture methodology was reported. All included articles were evaluated to identify any potential source of bias

Results Five papers were included in our qualitative analysis, for a total of 633 subjects. Compliance to per-protocol defined schedule of acupuncture sessions was high, ranging from 82 to $95.9 \%$. Most patients (70.6\%) were randomly allocated to receive acupuncture for its potential preventive effect on xerostomia. The large heterogeneity in study settings and clinical outcomes prevented from performing a cumulative quantitative analysis, thus no definitive recommendations can be provided. Conclusions Although shown to be feasible and safe, no firm evidence currently supports the use of acupuncture for the routine management of radiation-induced toxicity in HNSCC.
\end{abstract}

Keywords Head and neck cancer $\cdot$ Radiotherapy $\cdot$ Chemotherapy $\cdot$ Acupuncture $\cdot$ Toxicity

\section{Introduction}

Head and neck squamous cell carcinoma (HNSCC) encompasses a heterogeneous group of primary tumors with distinct histologic features and clinical behavior that arise from the oral cavity, oropharynx, hypopharynx, larynx and

Pierluigi Bonomo

bonomop@aou-careggi.toscana.it

1 Radiation Oncology, Azienda Ospedaliero-Universitaria Careggi, University of Florence, largo Brambilla 3, 50134 Florence, Italy

2 Cancer Risk Factors and Lifestyle Epidemiology Unit, Institute for Cancer Research, Prevention, and Clinical Network (ISPRO), Florence, Italy

3 Integrative Medicine Unit, Department of Anesthesia and Intensive Care, Azienda Ospedaliero-Universitaria Careggi, Florence, Italy nasopharynx, accounting for $6 \%$ of all malignant neoplasms [1]. At diagnosis, about $60 \%$ of patients have a loco-regionally advanced disease [2], potentially amenable to curatively intended management. Often in combination with cisplatinbased concurrent chemotherapy (CT), radiotherapy (RT) represents a mainstay of treatment for the majority of these

4 Department of Otorhinolaryngology-Head and Neck Surgery, Azienda Ospedaliero-Universitaria Careggi, Florence, Italy

5 Head and Neck Oncology and Robotic Surgery, Department of Experimental and Clinical Medicine, Azienda Ospedaliero-Universitaria Careggi, Florence, Italy

6 Maxillo Facial Surgery Unit, Azienda Ospedaliero-Universitaria Careggi, Florence, Italy

7 Fior Di Prugna Center for Complementary Medicine, Azienda USL Toscana Centro, Florence, Italy 
late-stage presentations, either as definitive modality or after surgery [3]. Alongside the recognition of the prominent role of RT in HNSCC management, the delivery of radiation has historically been challenged by the known complexity of head and neck anatomy, whereby it is paramount that radiation oncologists aim for the right balance between pursuing a tumoricidal effect and avoiding undue toxicity. The inadvertent dose accumulation to unaffected oral and pharyngeal mucosal surfaces, salivary glands, teeth, mandible, larynx, skin, and soft tissue of the neck, may be associated with the development of side effects, both short and long terms. Conventionally regarded per Common Terminology Criteria for Adverse Events (CTCAE) definition [4] as occurring within 3 months from the end of treatment, radiation-induced acute toxicity entails the onset of oral mucositis [5], dysphagia [6], dysgeusia [7], salivary impairment [8], and dermatitis [9], posing a threat to the safe delivery of prescribed RT dose intensity and patient's compliance. Late complications, such as xerostomia [10], trismus [11], osteoradionecrosis of the jaw [12], generalized fibrosis and lymphedema [13], muskuloskeletal disorders [14] and dental pathologies [15], may be prohibitive as well, with marked detriment to quality of life [16, 17] and social functioning [18] of HNSCC survivors. In addition, non-cancer-related mortality [19] can be considered as a potential treatment-induced late event, particularly in regards to aspiration pneumonia occurring after pharyngo-laryngeal dysfunction due to concurrent chemo-radiotherapy (CRT) [20, 21]. Thanks to its exquisite dose distribution allowing for improved sparing of organs at risk over conventional three-dimensional conformal techniques, Intensity-modulated radiation therapy (IMRT) was firmly established as standard practice for all patients with HNSCC [22]. Together with evidence-based demonstration of IMRT benefit [23], fostering multidimensional supportive care measures $[24,25]$ and implementing survivorship programs [26] have been more recently outlined as key care strategies. However, optimizing the management of patients developing radiation-induced acute and late toxicities is still an unmet need in head and neck cancer. Within the scope of complementary medicine the use of acupuncture for symptom control in oncology [27-29] has been increasingly advocated over the last two decades. Being recognized as an integral component of traditional Chinese medicine, acupuncture represents a complex nonpharmacologic intervention with a very favorable tolerability profile: briefly, it is characterized by the repeated application of thin metallic needles in discrete points of human anatomy ("acupoints") and their ensuing manipulation with the purpose to modulate brain regions involved in cognition and emotion. Currently, its potential mitigating effect is part of the therapeutic armamentarium for common systemic therapy-related side effects, such as nausea and vomiting [30], fatigue [31], chronic pain [32], peripheral neuropathy [33, 34], and arthralgia [35]. In the context of head and neck cancer, the results of a randomized phase 2 study published in 2010 [36] and subsequent observations [37, 38] lend support to the application of acupuncture mainly for symptoms occurring after neck dissection, such as shoulder pain and arm dysfunction. Concerning common radiation-induced side effects, the available evidence is sparse, hampering definitive recommendations in the clinic. Since the non-surgical management of locally advanced HNSCC is usually associated with a narrow therapeutic index and debilitating consequences, we aimed to address whether the use of acupuncture can be recommended for patients primarily treated with RT. In addition, we sought to analyze its potential impact on the occurrence and degree of severity of distinct radiationinduced side effects.

\section{Materials and methods}

In accordance with the Preferred Reporting Items for Systematic Reviews and Meta-Analyses (PRISMA) statement [39], a systematic review of the literature was conducted. Two electronic databases (Medline and Embase) were searched over a 10-year time frame (01/01/10 to 30/09/20). The MesH search strategy was as follows: PubMed search strategy, "head and neck radiotherapy AND acupuncture"("Head and Neck Neoplasms"[Mesh]) AND ("Radiotherapy"[Mesh]) AND "Acupuncture Therapy"[Mesh]; EMBASE search strategy, ('head and neck tumor'/exp OR 'head and neck tumor') AND ('radiotherapy'/exp OR radiotherapy) AND ('acupuncture'/exp OR acupuncture) AND [2010-2020]/ py AND [english]/lim. Case reports, case series with less than 20 patients, reviews and consensus statements were not included. Only full-text papers available in English could be evaluated. The reference lists of the reviewed articles were manually searched. Conference proceedings of main international conferences (ASCO, ASTRO, ESMO, and ESTRO) were also analyzed; however, data published in abstract form only were excluded. As a guidance for the search strategy, the following PICO criteria [40] were addressed:

\section{Population}

Patients undergoing a curatively intended, radiation-based treatment for histologically confirmed squamous cell carcinoma of the nasopharynx, oropharynx, larynx, hypopharynx and oral cavity represented the target population of our study. The use of CRT or RT as adjuvant therapy after primary surgery or as palliative treatment for recurrent/metastatic disease was not allowed. Subjects with HNSCC of any other primary anatomic location in the head and neck or non-squamous histologies were not eligible. 


\section{Intervention}

Reporting accurate information on the acupuncture methodology was mandatory upon inclusion of reviewed articles in our analysis. Details on the total number and individual duration of acupuncture sessions, the needle map insertion protocol, the use of sham technique as control arm and the degree of experience of board-certified acupuncturists were retrieved, whenever available. The timing of acupuncture application in respect to RT was also described, whether performed before, during or after its completion. Alternative techniques such as acupuncture-like transcutaneous electrical nerve stimulation (ALTENS) and combination acupuncture not complying with the Chinese traditional manipulation were not allowed.

\section{Comparators}

In view of their potential impact on the development of side effects, treatment-related features were defined as "comparators". In respect to RT, data on technique (3DCRT or IMRT), total dose, fractionation, dose correlates to relevant organs at risk such as parotid glands and pharyngeal constrictor muscles and treatment delays (elapsed days) were collected, whenever available. When systemic therapy was prescribed in combination with radiation, either one of the following options was categorized:

- 3-weekly cisplatin $\left(100 \mathrm{mg} / \mathrm{m}^{2}\right)$

- weekly cisplatin $\left(40 \mathrm{mg} / \mathrm{m}^{2}\right)$

- cetuximab (loading dose of $400 \mathrm{mg} / \mathrm{m}^{2}$, weekly dose of $250 \mathrm{mg} / \mathrm{m}^{2}$ )

- other

In all cases, information on the mean relative dose intensity (RDI, defined as the delivered percentage of the total planned dose) and median number of administered cycles were extrapolated, whenever available. In case induction chemotherapy was prescribed before RT, the same data were retrieved. In addition, qualitative descriptors of supportive measures (such as the use of prophylactic percutaneous endoscopic gastrostomy, early implementation of palliative care, hospitalization rate, etc.) were searched in the reviewed articles.

\section{Outcomes}

The potential impact of acupuncture on treatment-related side effects was differentiated based on their time of onset. By definition, acute toxicity referred to complications occurring during treatment or within 90 days from the end of radiation, whereas late toxicity to those developing thereafter. As per CTCAE [4], the following were the most common adverse events to be searched for: nausea, vomiting, dysgeusia, oral mucositis, radiation dermatitis, dysphagia, pain, fibrosis and xerostomia. The rates of any-grade and severe (G3-G4) toxicity were reported, if specified. The correlation between the use of acupuncture and health-related quality of life through standardized patient-reported outcomes was also explored, whenever available. Articles focused on surgical complications such as shoulder pain or dysfunction following neck dissection were not considered.

\section{Statistical analysis}

Baseline patient characteristics, disease and treatment features were summarized using descriptive statistics (mean, median and frequency distribution). In accordance with the Cochrane Review tool [41], all included articles were evaluated to identify any potential source of bias. In particular, the presence of bias was assessed in terms of risks of selection (random sequence generation), reporting (selective outcome reporting), performance (knowledge of the allocated interventions), detection (lack of precise definition and reliable method to detect and report the outcome), and attrition (deviations or handling of incomplete outcome data), respectively. Initially, we had planned to combine study-specific estimates of relative risks (risk ratio-RR or odds ratio-OR) for the effect of acupuncture on the incidence of treatmentrelated side effects into summary relative risks using random effects meta-analysis models, and to quantify the betweenstudies heterogeneity using the $I^{2}$ statistics. However, the small number and low comparability of eligible studies prevented us from performing this kind of approach.

\section{Results}

\section{Data collection and analysis}

An independent assessment of the reviewed literature was performed by two authors (PB and GS). The identified references were analyzed through a data collection sheet. Discrepancies were resolved by consensus with a third author (ID). By applying our prespecified search criteria, a total of 151 articles was retrieved (Fig. 1). After removing duplicates, 103 papers were screened through abstract assessment, 8 of whom qualified for full-text analysis. Homb et al. [42] performed a retrospective analysis of combination acupuncture for xerostomia on 16 patients, thus below the prespecified threshold of 20 subjects upon inclusion in our study. For the same reason, the interimreport [43] on first 15 patients enrolled in a randomized trial assessing the impact of acupuncture on acute toxicity during CRT was also excluded, whereas the publication on the controlled, randomized Rosetta trial [44] 


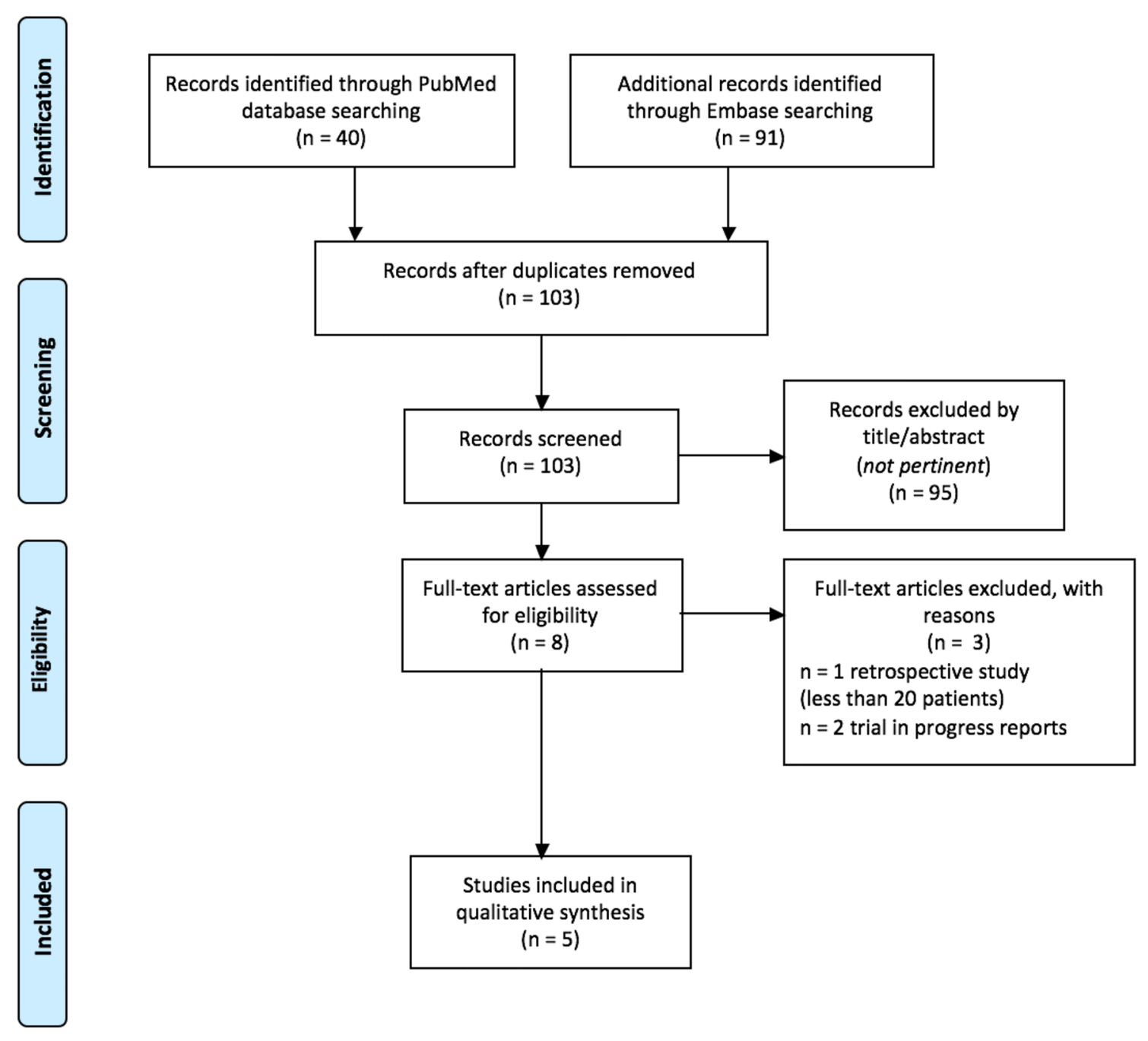

Fig. 1 Flow chart of literature search

had no patient information. Overall, 5 articles published between 2011 and 2019 fulfilled our inclusion criteria and were retained in the final analysis (Table 1). All were performed prospectively and had a randomized design, except for Braga's case-control cohort study [45]. In terms of Cochrane review tool assessment (Fig. 2), methods of random sequence generation and allocation of patients to treatment groups were specified in all included articles; therefore, a low risk of selection bias was assigned. No selective reporting of outcomes could also be detected. In addition, the attrition bias was deemed low as outcome data were found to be complete. In two studies [48, 49], a sham acupuncture (SA) arm was designed, allowing for blinding between treatment groups. Overall, despite the heterogeneity of the study population in the included articles and the variety of assessment scales for the main outcomes, an overall low risk of bias could be found.

\section{Patient, disease and treatment-related features}

A total of 633 patients was included in our analysis, of whom 328 (51.8\%), $313(49.4 \%)$ and 136 (21.4\%) received true acupuncture (TA), standard care (SC) and SA, respectively (Table 1). Of note, Simcock et al. [47] designed a randomized, cross-over phase 3 study, whereby all 144 enrolled subjects received TA, either anticipated (74/144, $51.3 \%)$ or followed $(70 / 14448.3 \%)$, by standard oral care. Most patients were male $(476 / 633,75.1 \%)$, with a mean age of 55.8 years (overall range across studies, 21-83). Despite the fact that in all studies, it was acknowledged that patients were in good clinical conditions and deemed candidate to receive a curatively intended treatment, no formal description of performance status could be retrieved from the included papers. In addition, no data were reported in terms of smoking exposure and pre-existing comorbidities. 


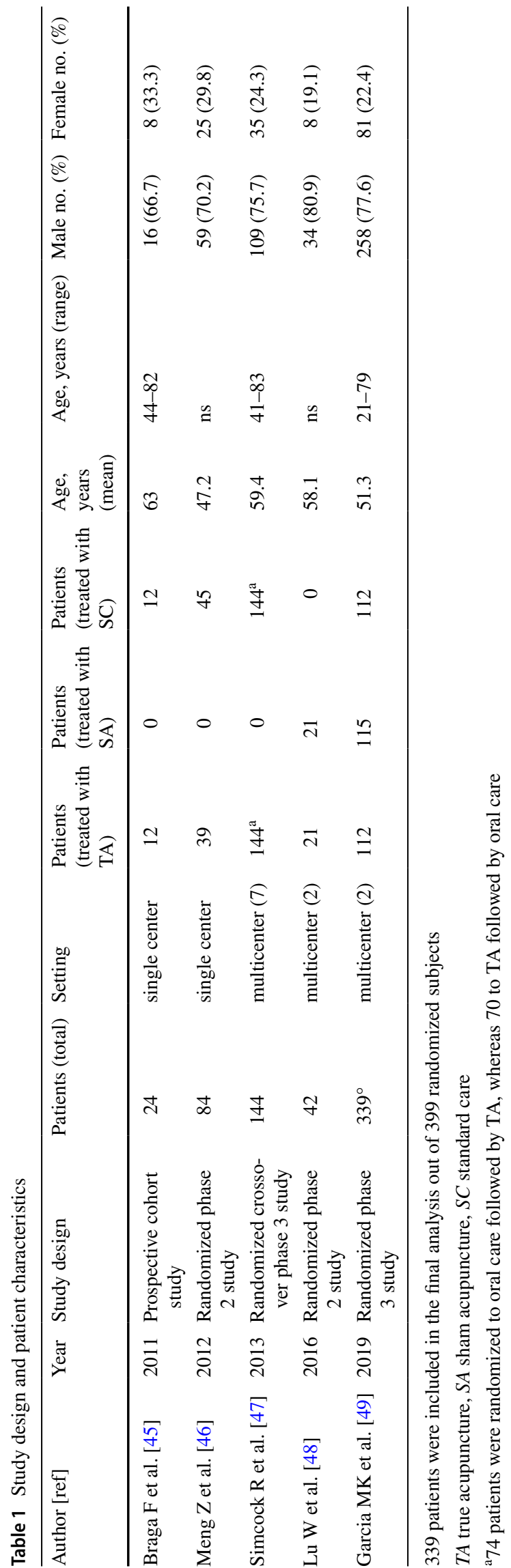

The two most common primary tumor sites were nasopharynx and oropharynx, with 314 (49.6\%) and 270 (42.6\%) cases each, respectively. No data could be found in regards to Human Papilloma Virus (HPV) status and Epstein Barr virus (EBV) detection. Although stage information was missing in two papers, most patients were treated for a locally advanced disease (combined stage III and IV, 403/484, 83.2\%) (Table 2). In regards to treatment characteristics, more than a third of the whole patient population was irradiated with outdated techniques (two dimensional and 3DCRT: $251 / 652,38.4 \%$ ), whereas IMRT was used in the two most recent works $[48,49]$ (Table 3). RT prescription did not differ significantly among the included studies or between arms, with a mean total dose ranging between 63.5 and 70.9 Gy delivered through a standard 6-7 week schedule. In respect to RT dose distribution to critical organs at risk and potential correlation with toxicity, some insight was provided in 3 papers. In Braga's [45] and Simcock's [47] works, it was reported that a significant portion of parotid glands was included in the designed RT fields. In spite of IMRT use, the optimal constraint (mean dose below $26 \mathrm{~Gy}$ ) for both parotids was not kept in over $60 \%$ of cases in Garcia's trial [49]. Notably, the mean dose to parotid glands was a stratification factor in this large, three-arm randomized study. Dosimetric data on oral cavity and pharyngeal constrictor muscles were not available. In view of the prevalent advanced disease stage of the whole sample, as expected most patients $(444 / 652,68 \%)$ received concurrent CT, although no information could be retrieved in terms of type of systemic agent, number of cycles and RDI. No data could be found on RT interruptions either. With the exception of the ARIX trial [47], where recruited patients had a minimum diseas-free interval of 18 months from RT completion, in the remaining 4 works acupuncture was mainly administered throughout a standard 6-7 week RT schedule, with variable frequency. In all studies, board-certified, experienced acupuncturists applied the needles, following well-defined protocols for about 20 min per session. Overall, the completion rate of acupuncture sessions was very high, ranging from $82 \%$ [46] to $95.9 \%$ [49]. No or very rare adverse events (i.e., mild discomfort, minor site bruise) related to acupuncture were reported, with a crude incidence far below $5 \%$ of the whole population. In two studies $[48,49]$ SA was performed in accordance with standardized methodology. In both trials, quality audits of both TA and SA were carried out per protocol. With the exception of the ARIX trial [47], where an educational approach of two group oral care sessions was envisaged before or after TA in both arms, in the remaining papers no specific intervention in the SC groups was attempted to minimize RT-related side effects, outside of routine management. Critically, no information on supportive care measures adopted during or after RT could be retrieved from the included studies. Overall, two additional 

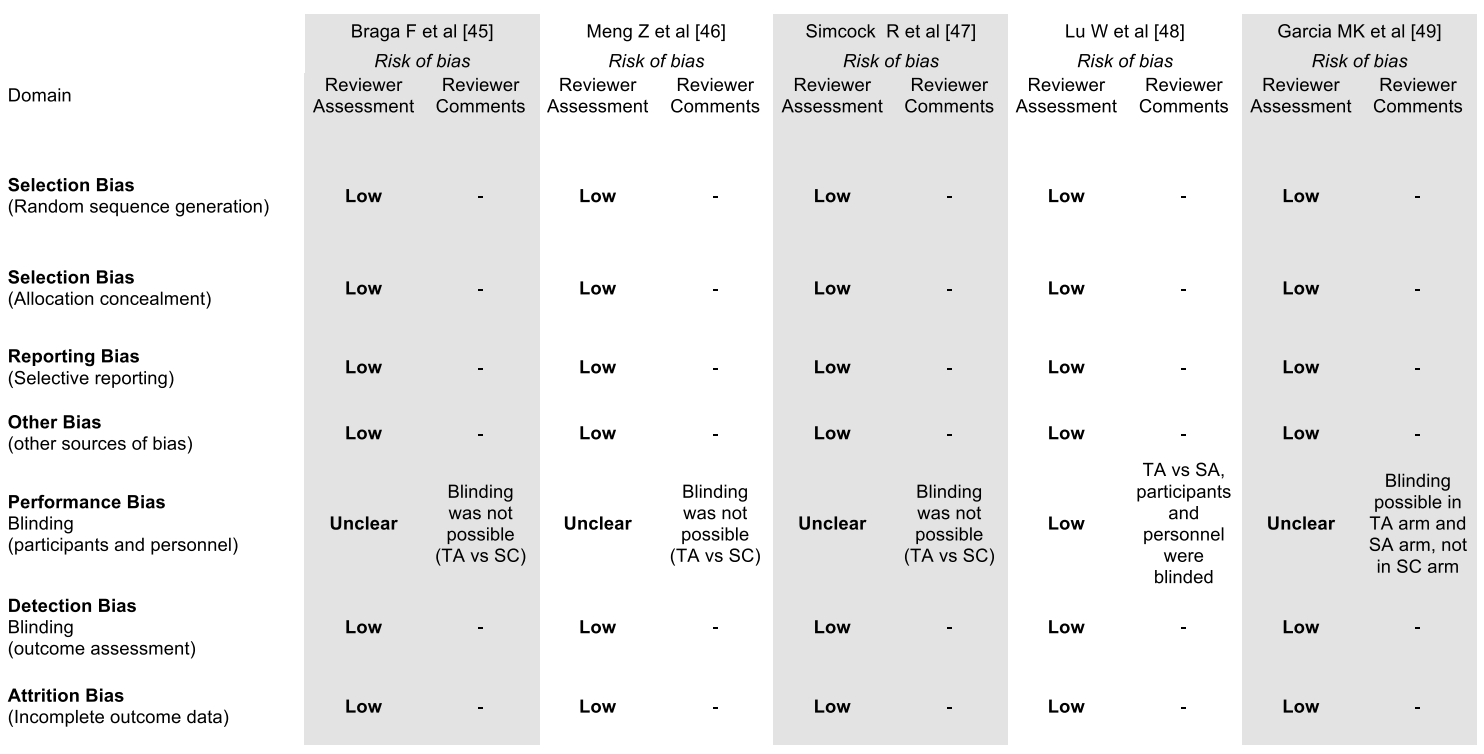

Fig. 2 Quality assessment according to the Cochrane review tool. $T A$ true acupuncture; $S A$ sham acupuncture; $S C$ standard care

limitations can be condensed from the analyzed studies: first, 74/652 patients (11.3\%) received some form of surgical manipulation before RT; however, as already mentioned, RT dose delivery was always in the curative range ( $>60 \mathrm{~Gy}$ ) for all cases. Second, the follow-up time was not uniformly completed, with $19 \%, 28 \%$ and $52.8 \%$ of missing data at predefined study timepoints in Lu's [48], Meng's [46] and Simcock's [47] works, respectively.

\section{Impact of acupuncture on radiation-induced toxicity}

The impact of acupuncture in mitigating radiation-induced side effects is summarized in Table 4 . The potential preventive effect of TA on acute xerostomia (by definition, occurring up to 3 months from RT completion) was addressed in 2 papers. In a small, non-randomized case-control study, Braga et al. [45] showed a significant difference in terms of objective sialometry measures on the last RT session. This finding was somewhat validated by Meng et al. [46], who observed a persistent effect on stimulated salivary flow rate up to 6 months after treatment (RR $0.62, p<0.003$ ). In addition, symptoms related to severe parotid dysfunction were significantly less likely (RR $0.63, p=0.019$ and RR 0.38 , $p=0.0018$ at 1 and 6 months, respectively) when patients had been exposed to TA during radiation. More recently, a large, dual-institution 3-arm randomized phase 3 trial [49] demonstrated that a preventive effect can be exerted on 1-year salivary dysfunction, as well. In comparison to SC, TA was associated with significantly fewer and less severe patient-reported xerostomia symptoms. However, subtle, marginally significant differences emerged when comparing the effect of TA with SA, in line with known concerns available from the literature [50] on the reliability of using placebo needles as control arm. In addition to the preventive role of TA on the occurrence of xerostomia, the multicenter ARIX trial [47] addressed its potential symptomatic efficacy in patients with chronic salivary impairment after 3DCRT. Although a significant degree of relief was demonstrated 9 weeks after TA in respect of specific patient-reported domains, such as "dry mouth" (RR 2.01, $p=0.031)$ and "sticky saliva" $\mathrm{RR}(1.67, p=0.048)$, no difference from an oral care educational session was observed in terms of overall quality of life and sialometry measures. Of note, the extremely variable timing between RT completion and randomization (median interval of 41 months, range 18-104 months) may have diluted the strenght of the intervention. In addition to xerostomia, a single small randomized phase 2 study [48] explored the potential preventive effect of TA on dysphagia 1 year after treatment. Other than demonstrating the feasibility of both TA and SA interventions, no significant difference emerged between the two arms in terms of dysphagia-related quality of life.

\section{Discussion}

To the best of our knowledge, this is the first systematic review of the literature based on PICO criteria aimed to evaluate the role of acupuncture in the management of radiationinduced toxicity in head and neck cancer. The significant burden in terms of long-lasting side effects [51] and quality of life impairment $[52,53]$ inflicted by standard CRT on patients with HNSCC call into question the potential need to integrate non-pharmacologic supportive care measures 


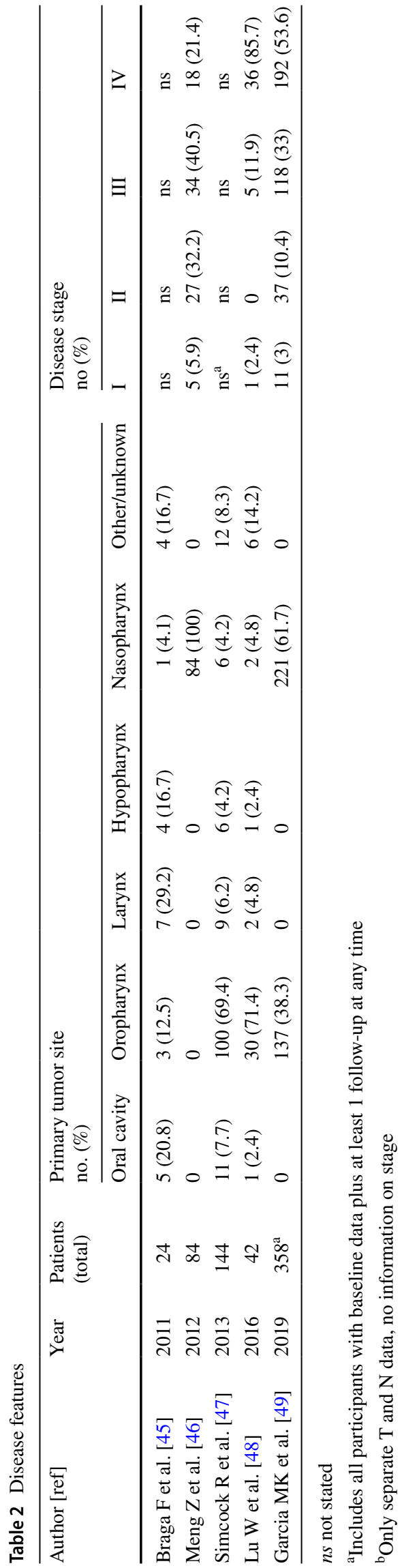

for symptom control. In view of the growing consideration of acupuncture within the therapeutic armamentarium [54-56] for common systemic therapy related toxicities, and in recognition of the limited data [57] available in respect to radiation therapy, we sought to analyze the literature in the most rigorous way, aiming to provide evidence-based recommendations for clinical practice in head and neck cancer. Following our prespecified search strategy, only a limited number of articles qualified for qualitative analysis. We had initially planned to pool the study-specific measures of association between acupuncture and the outcomes of interest (radiation-induced toxicities) into summary estimates using random-effects meta-analysis models. However, this was not possible because of the too limited number of available estimates for some endpoints; the large heterogeneity in terms of study settings and, in particular, in the way the outcomes were defined and measured; and the variability in reporting the measures of association with acupuncture. Therefore, the salient features and the main findings of the studies included in the systematic review were presented in tables and commented narratively in the text, whereas no formal statistical analysis could be conducted. Over $70 \%$ of patients (447/633) included in our work were randomly allocated to receive acupuncture throughout RT or CRT to evaluate its potential preventive effect on xerostomia. Overall, the most relevant common finding in both Meng's [46] and Garcia's [49] papers was that the delivery of TA was significantly associated with less severe patient-reported symptoms of salivary impairment (by definition, a score of 30 or more with the validated 8-item Xerostomia Questionnaire) compared with standard supportive measures up to 1 year from the end of treatment. In addition, in terms of preserving stimulated salivary flow rate, similar results were reported by Braga [45] and Meng [46]. As already anticipated, caution should be advised when addressing these 3 studies [45, 46, 49] together, keeping in mind their low comparability due to large differences in terms of timepoints of assessment, methodology, limited follow-up time and heterogeneity in patient and treatment characteristics, in particular RT technique. In this regard, extensive radiobiological models [58] underpinned the critical dependence of parotid gland damage from radiation dose distribution. In the pivotal phase 3 randomized Parsport trial [23], a mean difference of 24.5 (99\% CI -4.3 to $53.2 ; 59.3$ to 34.8 , $p<0.0001$ ) was observed 24 months after RT on the EORTC HN35 dry mouth subscale score in favour of patients treated with IMRT over 3DCRT. Selective sparing of stem cells in parotid sub-regions [59], pre-treatment functional characterization through quantitative imaging [60] and machine learning applications [61] are active areas of research for improving the technical capability of xerostomia prevention. For this purpose, no pharmacological interventions are currently supported in the clinic with sufficient evidence 


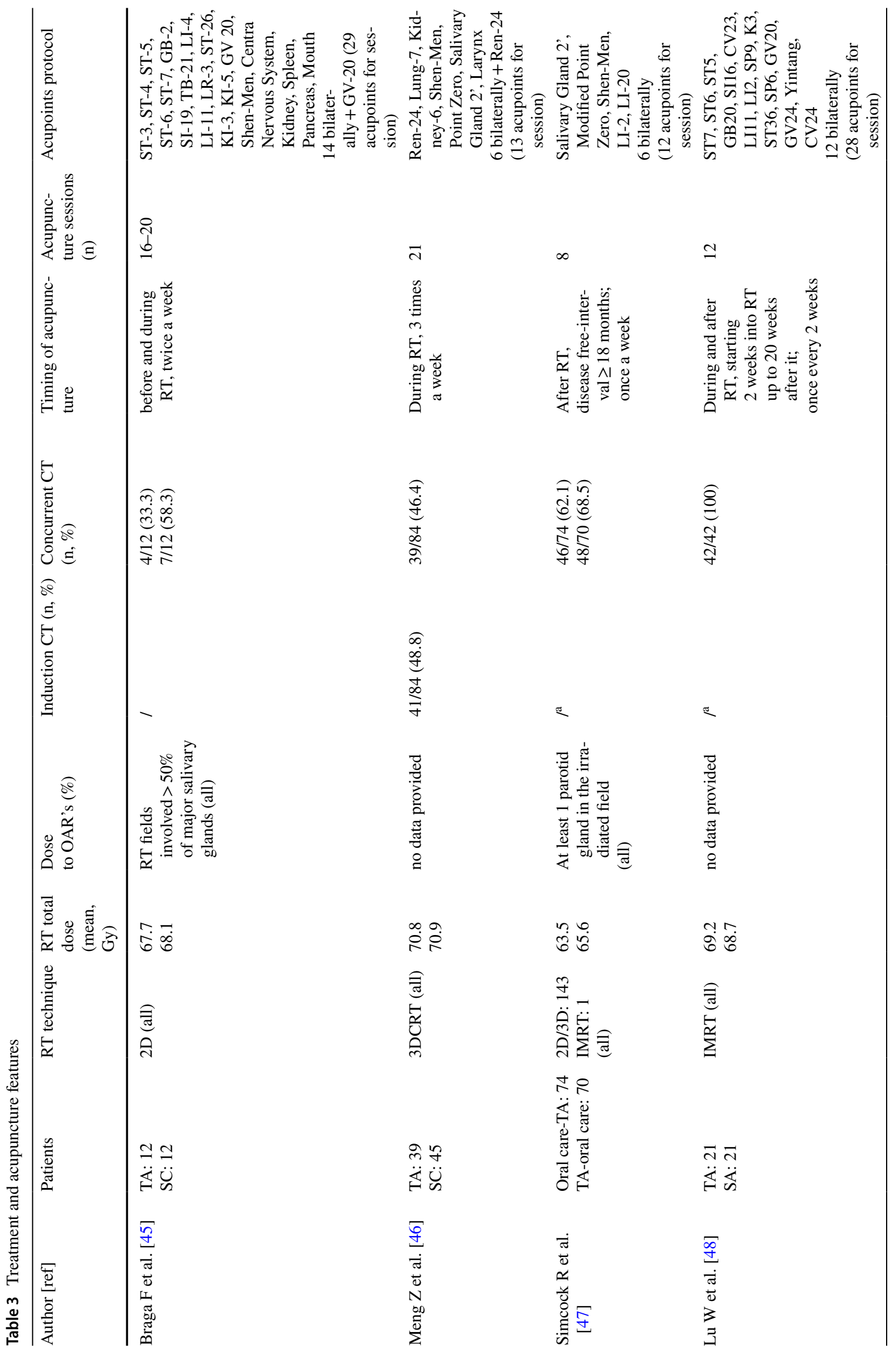




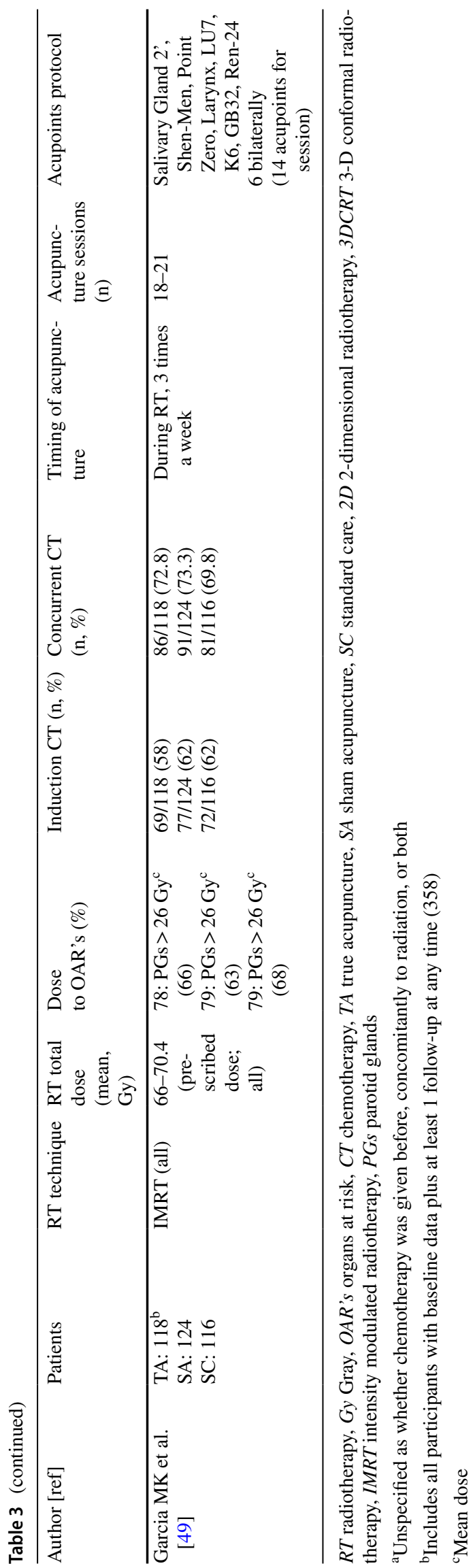

[62-64]. Albeit not further replicated in prospective studies, the results reported by Simcock et al. [47] lend support to the potential symptomatic efficacy of acupuncture on radiationinduced late salivary impairment, with significant improvement on the rate of patient-reported severe dry mouth (OR $2.0, p=0.031$ ), sticky saliva (OR 1.67, $p=0.048$ ), need to sip to swallow food (OR 2.08, $p=0.011$ ), and in waking up at night to drink (OR 1.71, $p=0.013$ ), over standard oral care. From a mechanistic perspective, limited data are available [27] to justify the relieving effect of acupuncture on xerostomia. In a prospective trial, 20 healthy volunteers were randomized to receive TA or SA in a blinded fashion. By assessing the correlation between needle manipulation at L1-L2 acupoint (radial side of the non-dominant hand), stimulated salivary flow rate and functional magnetic resonance imaging, Deng et al. [65] demonstrated a higher salivation (mean difference of $0.34 \mathrm{~g}$ ) obtained with TA over SA. In addition, a fair correlation between saliva production and neuroimaging activation of specific neuronal circuitry (parietal operculum, rolandic operculum, frontal operculum and insula, bilaterally) was also shown ( 0.63 coefficient at linear regression). The existence of a different pattern of neuroimaging activation induced by specific acupoint stimulation for the head and neck area was corroborated by further observations $[66,67]$ in non-oncologic patients. In analogy to other symptoms, it was hypothesized that the mitigating effect of acupuncture on xerostomia may result from its modulation of systemic inflammatory cascade and of parasympathetic and sympathetic balance at a glandular level [68, 69].

As demonstrated by our study results, other than xerostomia, very limited data [43, 48, 70-72] are currently available on the role of acupuncture in respect to other treatmentrelated side effects in HNSCC. In particular, there is little evidence [48] regarding the potential preventive effect on radiation-induced dysphagia. At diagnosis, most patients with locally advanced HNSCC already suffer from a variable degree of swallowing impairment [73]: its baseline assessment and management during therapy are extremely important factors with potential implications on the therapeutic success [74]. The adoption of preventive measures such as behavioral swallowing interventions may be helpful, albeit associated with a generally modest rate of patient adherence [75] and unclear impact on patients' quality of life [76], being supported by low-level evidence [77]. Currently, the most reproducible way to try to mitigate the effects of treatment on the swallowing function resides in the implementation of swallowing-sparing IMRT. In last 15 years, a large body of evidence $[6,78,79]$ was published on the correlation between radiation dose to pharyngeal constrictor muscles and the risk of developing acute and late swallowing impairment. No pharmacologic interventions aimed at preventing radiation-induced dysphagia can be recommended in 


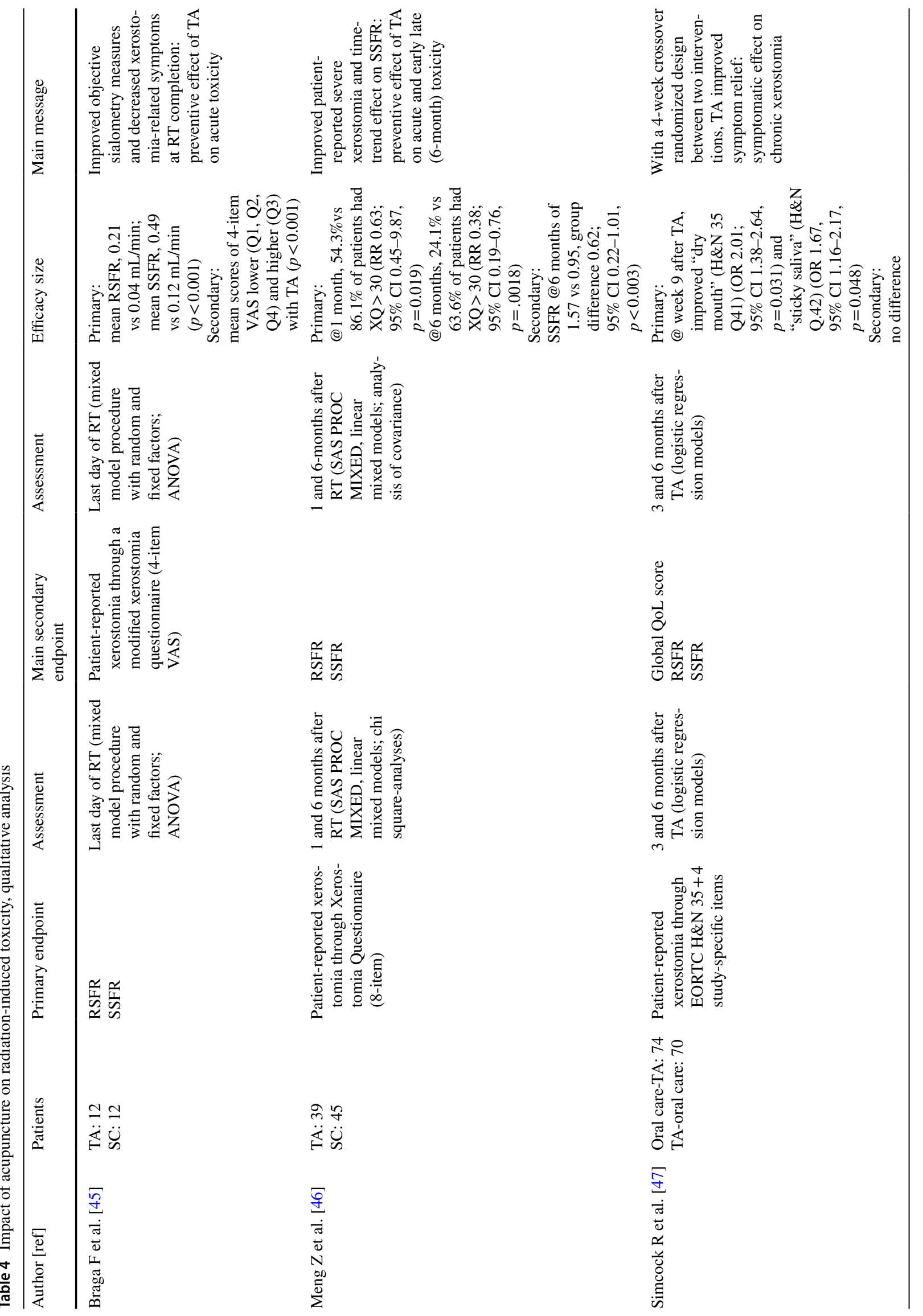




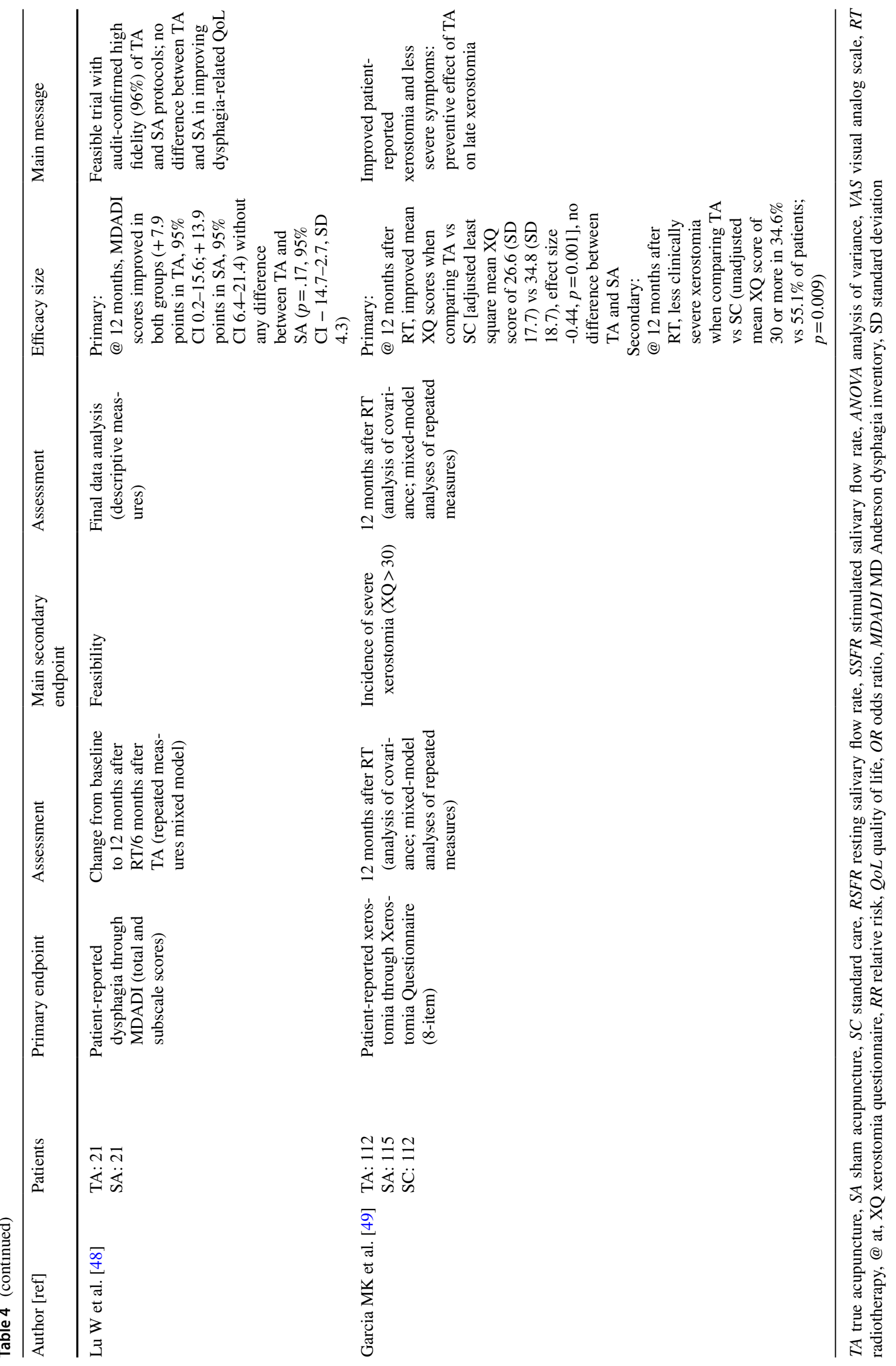


the clinic. With the aim to demonstrate a protective effect of TA on patient-reported dysphagia in terms of M.D. Anderson Dysphagia Inventory (MDADI) [80] change from baseline to 1 year after treatment, Lu et al. [48] were not able to show any difference between TA and SA arms (improvement from baseline: +7.9 points in TA arm, 95\% CI 0.2-15.6, and +13.9 points in SA arm, 95\% CI 6.4-21.4; $p=0.17$ for the comparison, $95 \% \mathrm{CI}-14.7-2.7)$. However, only $34 / 42$ patients $(81 \%)$ completed the study follow-up visits, with a potential imbalance in terms of RT total dose delivery between the two groups (70 Gy delivered in 90 and 76\% of TA and SA groups, respectively). Throughout CRT, the onset of dysphagia should be considered as a critical component of a multifactorial painful syndrome [81, 82]: in view of the known analgesic effect of acupuncture in cancer [32] and preclinical data [83,84], additional evidence is required to elucidate its preventive potential on irradiated swallowing structures. For the time being, state-of-the-art parotid and swallowing-sparing IMRT must be regarded as standard of care in everyday's clinical practice.

Overall, the results of our study reinforce the notion that acupuncture is feasible in conjunction with anti-cancer treatment even if intensive as concurrent chemo-radiation can be, being associated with a very favorable toxicity profile. As reflected by our data, there's increasing interest in applying acupuncture in head and neck cancer [38, 72, 85]. Although a detailed description is not within the scope of this work, our findings indirectly confirm the shortcomings bound with performing clinical research in the field of acupuncture [50, 86-88]. In spite of the methodological rigorousness based on a PICO-literature search, the large heterogeneity found in the retrieved data restrained us from providing any definitive recommendation on the role of acupuncture in the field of HNSCC. We acknowledge that focusing on papers published in languages other than English (i.e., Chinese) may represent a limitation of our study, as well as the exclusion of results obtained with ALTENS [89-91].

\section{Conclusions}

In the context of locally advanced HNSCC, no evidence currently supports the routine use of acupuncture for the majority of acute and late radiation-induced side effects. Although shown to be feasible and safe in a single pilot randomized sham-controlled trial, no data currently justify its clinical application for the prevention of dysphagia. The large heterogeneity and low comparability of studies highlighting the impact of acupuncture on acute and late xerostomia don't allow to provide any definitive recommendation in this regard. Further well-designed, controlled clinical trials should be warranted.
Funding Open access funding provided by Università degli Studi di Firenze within the CRUI-CARE Agreement.

Data availability Data supporting the findings of this study are available in the article or supplementary files.

Code availability Not applicable.

\section{Declarations}

Conflict of interest None to be declared.

Ethical approval Obtained by local committee.

Consent to participate Obtained by single studies.

Consent for publication Obtained by single studies.

Open Access This article is licensed under a Creative Commons Attribution 4.0 International License, which permits use, sharing, adaptation, distribution and reproduction in any medium or format, as long as you give appropriate credit to the original author(s) and the source, provide a link to the Creative Commons licence, and indicate if changes were made. The images or other third party material in this article are included in the article's Creative Commons licence, unless indicated otherwise in a credit line to the material. If material is not included in the article's Creative Commons licence and your intended use is not permitted by statutory regulation or exceeds the permitted use, you will need to obtain permission directly from the copyright holder. To view a copy of this licence, visit http://creativecommons.org/licenses/by/4.0/.

\section{References}

1. Siegel RL, Miller KD, Jemal A (2020) Cancer statistics, 2020. Ca Cancer J Clin 70:7-30

2. Chow LQM (2020) Head and neck cancer. N Eng J Med 382:60-72

3. NCCN guidelines (2021) Head and neck cancers, version 1.2021. Available at: http://www.nccn.org. Accessed 13 June 2021

4. Common Terminology Criteria for Adverse Events (CTCAE). Available at: http://www.ctep.cancer.gov. Accessed 13 June 2021

5. Trotti A, Bellm LA, Epstein JB et al (2003) Mucositis incidence, severity and associated outcomes in patients with head and neck cancer receiving radiotherapy with or without chemotherapy: a systematic literature review. Radiother Oncol 66:253-262

6. Eisbruch A, Schwartz M, Rasch C et al (2004) Dysphagia and aspiration after chemoradiotherapy for head-and-neck cancer: which anatomic structures are affected and can they be spared by IMRT? Int J Radiat Oncol Biol Phys 60:1425-1439

7. Hovan AJ, Williams PM, Stevenson-Moore P et al (2010) A systematic review of dysgeusia induced by cancer therapies. Support Care Cancer 18:1081-1087

8. Belli ML, Scalco E, Sanguineti G et al (2014) Early changes of parotid density and volume predict modifications at the end of therapy and intensity of acute xerostomia. Strahlentherapie Und Onkol 190:1001-1007

9. Hymes SR, Strom EA, Fife C (2006) Radiation dermatitis: clinical presentation, pathophysiology, and treatment 2006. J Am Acad Dermatol 54:28-46 
10. Eisbruch A, Ten Haken RK, Kim HM, Marsh LH, Ship JA (1999) Dose, volume, and function relationships in parotid salivary glands following conformal and intensity-modulated irradiation of head and neck cancer. Int J Radiat Oncol Biol Phys 45:577-587

11. Dijkstra PU, Kalk WWI, Roodenburg JLN (2004) Trismus in head and neck oncology: a systematic review. Oral Oncol 40:879-889

12. Caparrotti F, Huang SH, Lu L et al (2017) Osteoradionecrosis of the mandible in patients with oropharyngeal carcinoma treated with intensity-modulated radiotherapy. Cancer 123:3691-3700

13. Deng J, Wulff-Burchfield EM, Murphy BA (2019) Late soft tissue complications of head and neck cancer therapy: lymphedema and fibrosis. J Natl Cancer Inst - Monogr 2019:63-71

14. Ghiam MK, Mannion K, Dietrich MS, Stevens KL, Gilbert J, Murphy BA (2017) Assessment of musculoskeletal impairment in head and neck cancer patients. Support Care Cancer 25:2085-2092

15. Buglione M, Cavagnini R, Di Rosario F et al (2016) Oral toxicity management in head and neck cancer patients treated with chemotherapy and radiation: xerostomia and trismus (Part 2). Literature review and consensus statement. Crit Rev Oncol Hematol 102:47-54

16. Ringash J (2015) Survivorship and quality of life in head and neck cancer. J Clin Oncol 33:3322-3327

17. Kam D, Salib A, Gorgy G et al (2015) Incidence of suicide in patients with head and neck cancer. JAMA Otolaryngol - Head Neck Surg 141:1075-1081

18. Massa ST, Osazuwa-Peters N, Adjei Boakye E, Walker RJ, Ward GM (2019) Comparison of the financial burden of survivors of head and neck cancer with other cancer survivors. JAMA Otolaryngol - Head Neck Surg 145:239-249

19. Mell LK, Dignam JJ, Salama JK et al (2010) Predictors of competing mortality in advanced head and neck cancer. J Clin Oncol 28:15-20

20. Orlandi E, Miceli R, Infante G et al (2019) Predictors of patientreported dysphagia following imrt plus chemotherapy in oropharyngeal cancer. Dysphagia 34:52-62

21. Forastiere AA, Zhang Q, Weber RS et al (2013) Long-term results of RTOG 91-11: a comparison of three nonsurgical treatment strategies to preserve the larynx in patients with locally advanced larynx cancer. J Clin Oncol 31:845-852

22. Grégoire V, Langendijk JA, Nuyts S (2015) Advances in radiotherapy for head and neck cancer. J Clin Oncol 33:3277-3284

23. Nutting CM, Morden JP, Harrington KJ et al (2011) Parotidsparing intensity modulated versus conventional radiotherapy in head and neck cancer (PARSPORT): a phase 3 multicentre randomised controlled trial. Lancet Oncol 12:127-136

24. Murphy BA, Deng J (2015) Advances in supportive care for late effects of head and neck cancer. J Clin Oncol 33:3314-3321

25. Bonomo P, Paderno A, Mattavelli D, Zenda S, Cavalieri S, Bossi P (2019) Quality assessment in supportive care in head and neck cancer. Front Oncol 9:1-9

26. Cohen EEW, LaMonte SJ, Erb NL et al (2016) American cancer society head and neck cancer survivorship care guideline. CA Cancer J Clin 66:203-239

27. Zia FZ, Olaku O, Bao T et al (2017) The National cancer institute's conference on acupuncture for symptom management in oncology: state of the science, evidence, and research gaps. J Natl Cancer Inst Monogr. https://doi.org/10.1093/jncimonogr aphs/lgx005

28. Kay Garcia M, Mcquade J, Haddad R et al (2013) Systematic review of acupuncture in cancer care: a synthesis of the evidence. J Clin Oncol 31:952-960

29. Wu X, Chung VCH, Hui EP et al (2015) Effectiveness of acupuncture and related therapies for palliative care of cancer: overview of systematic reviews. Sci Rep 5:1-15
30. Li QW, Li QW, Yu MW et al (2020) Efficacy of acupuncture in the prevention and treatment of chemotherapy-induced nausea and vomiting in patients with advanced cancer: a multi-center, single-blind, randomized, sham-controlled clinical research. Chin Med (United Kingdom) 15:1-11

31. Mao JJ, Farrar JT, Bruner D et al (2014) Electroacupuncture for fatigue, sleep, and psychological distress in breast cancer patients with aromatase inhibitor-related arthralgia: a randomized trial. Cancer 120:3744-3751

32. He Y, Guo X, May BH et al (2020) Clinical evidence for association of acupuncture and acupressure with improved cancer pain: a systematic review and meta-analysis. JAMA Oncol 6:271-278

33. Greenlee H, Crew KD, Capodice J et al (2016) Randomized shamcontrolled pilot trial of weekly electro-acupuncture for the prevention of taxane-induced peripheral neuropathy in women with early stage breast cancer. Breast Cancer Res Treat 156:453-464

34. Loprinzi CL, Lacchetti C, Bleeker J et al (2020) Prevention and management of chemotherapy-induced peripheral neuropathy in survivors of adult cancers: ASCO guideline update. J Clin Oncol 38:3325-3348

35. Hershman DL, Unger JM, Greenlee H et al (2018) Effect of acupuncture vs sham acupuncture or waitlist control on joint pain related to aromatase inhibitors among women with early-stage breast cancer a randomized clinical trial. JAMA-J Am Med Assoc 320:167-176

36. Pfister DG, Cassileth BR, Deng GE et al (2010) Acupuncture for pain and dysfunction after neck dissection: results of a randomized controlled trial. J Clin Oncol 28:2565-2570

37. Deganello A, Battat N, Muratori E et al (2016) Acupuncture in shoulder pain and functional impairment after neck dissection: a prospective randomized pilot study. Laryngoscope 126:1790-1795

38. Matovina C, Birkeland AC, Zick S, Shuman AG (2017) Integrative medicine in head and neck cancer. Otolaryngol Head Neck Surg 156:228-237

39. Liberati A, Altman DG, Tetzlaff J et al (2009) The PRISMA statement for reporting systematic reviews and meta-analyses of studies that evaluate health care interventions: explanation and elaboration. J Clin Epidemiol 62:e1-34

40. Aslam S, Emmanuel P (2010) Formulating a researchable question: a critical step for facilitating good clinical research. Indian J Sex Transm Dis AIDS 31:47-50

41. Higgins JPT, Altman DG (2008) Assessing risk of bias in included studies. Cochrane handbook for systematic reviews of interventions, 5th edn. Wiley, pp 187-241

42. Homb KA, Wu H, Tarima S, Wang D (2014) Improvement of radiation-induced xerostomia with acupuncture: a retrospective analysis. J Arthe 2:34-38

43. Dymackova R, Kazda T, Slavik M, Selingerova I, Slampa P, Slama O (2020) Acupuncture in the treatment of acute toxicity during and after head and neck cancer radiotherapy: interim analysis of randomized prospective open-label trial. Biomed Pap Med Fac Univ Palacky Olomuc Czech Repub 164:454-460

44. Asadpour R, Kessel KA, Bruckner T, Sertel S, Combs SE (2017) Randomized study exploring the combination of radiotherapy with two types of acupuncture treatment (ROSETTA): study protocol for a randomized controlled trial. Trials 18:398

45. Braga FP, Lemos Junior CA, Alves FA, Migliari DA (2011) Acupuncture for the prevention of radiation-induced xerostomia in patients with head and neck cancer. Braz Oral Res 25:180-185

46. Meng Z, Garcia MK, Hu C et al (2012) Randomized controlled trial of acupuncture for prevention of radiation-induced xerostomia among patients with nasopharyngeal carcinoma. Cancer 118:3337-3344 
47. Simcock R, Fallowfield L, Monson K et al (2013) Arix: a randomised trial of acupuncture $\mathrm{V}$ oral care sessions in patients with chronic xerostomia following treatment of head and neck cancer. Ann Oncol 24:776-783

48. Lu W, Wayne PM, Davis RB et al (2016) Acupuncture for chemoradiation therapy-related dysphagia in head and neck cancer: a pilot randomized sham-controlled trial. Oncologist 21:1522-1529

49. Garcia MK, Meng Z, Rosenthal DI et al (2019) Effect of true and sham acupuncture on radiation-induced xerostomia among patients with head and neck cancer: a randomized clinical trial. JAMA Netw Open 2:e1916910

50. Zhang LL, Chu Q, Wang S, Lai H, Xie BB (2016) Is sham acupuncture as effective as traditional Chinese acupuncture? It's too early to say. Chin J Integr Med 22:483-489

51. Machtay M, Moughan J, Trotti A et al (2008) Factors associated with severe late toxicity after concurrent chemoradiation for locally advanced head and neck cancer: an RTOG analysis. J Clin Oncol 26:3582-3589

52. Langendijk JA, Doornaert P, Verdonck-de Leeuw IM, Leemans CR, Aaronson NK, Slotman BJ (2008) Impact of late treatment-related toxicity on quality of life among patients with head and neck cancer treated with radiotherapy. J Clin Oncol 26:3770-3776

53. Vainshtein JM, Moon DH, Feng FY, Chepeha DB, Eisbruch A, Stenmark MH (2015) Long-term quality of life after swallowing and salivary-sparing chemo-intensity modulated radiation therapy in survivors of human papillomavirus-related oropharyngeal cancer. Int J Radiat Oncol Biol Phys 91:925-933

54. Garcia MK, McQuade J, Lee R, Haddad R, Spano M, Cohen L (2014) Acupuncture for symptom management in cancer care: an update. Curr Oncol Rep 16:418

55. Shalom-Sharabi I, Samuels N, Lev E et al (2017) Impact of a complementary/integrative medicine program on the need for supportive cancer care-related medications. Supp Care Cancer 25:3181-3190

56. Acupuncture (PDQ ${ }^{\circledR)}$ ): Health Professional Version. $P D Q$ Cancer Inf Summ 2002-2020 http://www.ncbi.nlm.nih.gov/pubmed/ 26389159 Accessed 1 October 2020

57. Asadpour R, Meng Z, Kessel KA, Combs SE (2016) Use of acupuncture to alleviate side effects in radiation oncology: current evidence and future directions. Adv Radiat Oncol 1(4):344-350

58. Deasy JO, Moiseenko V, Marks L, Chao KS, Nam J, Eisbruch A (2010) Radiotherapy dose-volume effects on salivary gland function. Int J Radiat Oncol Biol Phys 76(3 Suppl.):S58-63

59. van Luijk P, Pringle S, Deasy JO et al (2015) Sparing the region of the salivary gland containing stem cells preserves saliva production after radiotherapy for head and neck cancer. Sci Transl Med 7:305ra147

60. Desideri I, Loi M, Francolini G, Becherini C, Livi L, Bonomo P (2020) Application of radiomics for the prediction of radiationinduced toxicity in the IMRT era: current state-of-the-art. Front Oncol 10:1708

61. Gabrys HS, Buettner F, Sterzing F, Hauswald H, Bangert M (2018) Design and selection of machine learning methods using radiomics and dosiomics for normal tissue complication probability modeling of xerostomia. Front Oncol 8:35

62. Bardet E, Martin L, Calais G et al (2011) Subcutaneous compared with intravenous administration of amifostine in patients with head and neck cancer receiving radiotherapy: final results of the GORTEC2000-02 phase III randomized trial. J Clin Oncol 29:127-133

63. Riley P, Glenny AM, Hua F, Worthington HV (2017) Pharmacological interventions for preventing dry mouth and salivary gland dysfunction following radiotherapy. Cochrane Database Syst Rev 7:CD012744
64. Lee MG, Freeman AR, Roos DE, Milner AD, Borg MF (2019) Randomized double-blind trial of amifostine versus placebo for radiation-induced xerostomia in patients with head and neck cancer. J Med Imaging Radiat Oncol 63:142-150

65. Deng G, Hou BL, Holodny AI, Cassileth BR (2008) Functional magnetic resonance imaging (fMRI) changes and saliva production associated with acupuncture at LI-2 acupuncture point: a randomized controlled study. BMC Complement Altern Med $8: 37$

66. Kong SP, Tan QW, Liu Y et al (2015) Specific correlation between the hegu point (LI4) and the orofacial part: evidence from an fMRI study. Evid Based Complement Alternat Med 2015:585493

67. Li LL, Liu XW, Wu F, Tong DC, Ye LP, Tao HX et al (2019) Electroacupuncture stimulation of language-implicated acupoint tongli (HT 5) in healthy subjects: an fMRI evaluation study. Chin J Integr Med 24:822-829

68. Lim HD, Kim MH, Lee CY, Namgung U (2016) Anti-inflammatory effects of acupuncture stimulation via the vagus nerve. PLoS ONE 11:e0151882

69. Park JY, Namgung UK (2018) Electroacupuncture therapy in inflammation regulation: current perspectives. J Inflamm Res 11:227-237

70. Djaali W, Simadibrata CL, Nareswari I (2020) Acupuncture therapy in post-radiation head-and-neck cancer with dysgeusia. Med Acupunct 32:157-162

71. Gane EM, Michaleff ZA, Cottrell MA et al (2017) Prevalence, incidence, and risk factors for shoulder and neck dysfunction after neck dissection: a systematic review. Eur J Surg Oncol 43:1199-1218

72. Almeida KAM, Rocha AP, Carvas N, Pinto ACPN (2020) Rehabilitation interventions for shoulder dysfunction in patients with head and neck cancer: systematic review and meta-analysis. Phys Ther 100(11):1997-2008

73. Paleri V, Roe WG, Strojan P et al (2014) Strategies to reduce longterm postchemoradiation dysphagia in patients with head and neck cancer: an evidence-based review. Head Neck 36:431-443

74. Schindler A, Denaro N, Russi EG et al (2015) Dysphagia in head and neck cancer patients treated with radiotherapy and systemic therapies: literature review and consensus. Crit Rev Oncol Hematol 96:372-384

75. Krekeler BN, Broadfoot CK, Johnson S, Connor NP, Rogus-Pulia N (2018) Patient adherence to dysphagia recommendations: a systematic review. Dysphagia 33:173-184

76. Messing BP, Ward EC, Lazarus C et al (2019) Establishing a multidisciplinary head and neck clinical pathway: an implementation evaluation and audit of dysphagia-related services and outcomes. Dysphagia 34:89-104

77. Greco E, Simic T, Ringash J, Tomlinson G, Inamoto Y, Martino R (2018) Dysphagia treatment for patients with head and neck cancer undergoing radiation therapy: a meta-analysis review. Int J Radiat Oncol Biol Phys 101:421-444

78. Szczesniak MM, Maclean J, Zhang T, Graham PH, Cook IJ (2014) Persistent dysphagia after head and neck radiotherapy: a common and under-reported complication with significant effect on noncancer-related mortality. Clin Oncol 26:697-703

79. Christianen MEMC, Langendijk JA, Westerlaan HE, Van De Water TA, Bijl HP (2011) Delineation of organs at risk involved in swallowing for radiotherapy treatment planning. Radiother Oncol 101:394-402

80. Chen AY, Frankowshi R, Bishop-Leone J et al (2001) The development and validation of a dysphagia-specific quality-of-life questionnaire for patients with head and neck cancer: the M. D. Anderson dysphagia inventory. Arch Otolaryngol-Head Neck Surg 127:870-876

81. Bossi P, Giusti R, Tarsitano A et al (2019) The point of pain in head and neck cancer. Crit Rev Oncol Hematol 138:51-59 
82. Bossi P, Ghiani M, Argenone A, Depenni R (2020) Is pain part of a systemic syndrome in head and neck cancer? Support Care Cancer 28:451-459

83. Kim S, Zhang X, O'Buckley SC, Cooter M, Park JJ, Nackley AG (2018) Acupuncture resolves persistent pain and neuroinflammation in a mouse model of chronic overlapping pain conditions. $\mathrm{J}$ Pain 19(12):1384.e1-1384.e14

84. Li Y, Fang Z, Gu N et al (2019) Inhibition of chemokine CX3CL1 in spinal cord mediates the electroacupuncture-induced suppression of inflammatory pain. J Pain Res 12:2663-2672

85. Nguyen CT, Taw MB, Wang MB (2018) Integrative care of the patient with head and neck cancer. Laryngoscope Investig Otolaryngol 3:364-371

86. Karst M, Li C (2019) Acupuncture-a question of culture. JAMA Netw Open 2:e1916929

87. Liu W, Qdaisat A, Lopez G et al (2020) Association between acupoint selection, target symptoms, and traditional Chinese medicine diagnosis in real-time clinical practice in a comprehensive cancer center. Integr Cancer Ther 19:1534735420928490

88. Liu W, Cohen L (2020) Overcoming barriers for clinical research of acupuncture. Med Acupunct 32:348-3551
89. Wong RKW, James JL, Sagar S et al (2012) Phase 2 results from radiation therapy oncology group study 0537: a phase $2 / 3$ study comparing acupuncture-like transcutaneous electrical nerve stimulation versus pilocarpine in treating early radiation-induced xerostomia. Cancer 118:4244-4252

90. Wong RKW, Deshmukh S, Wyatt G et al (2015) Acupuncturelike transcutaneous electrical nerve stimulation versus pilocarpine in treating radiation-induced xerostomia: results of RTOG 0537 phase 3 study. Int J Radiat Oncol Biol Phys 92:220-227

91. Iovoli AJ, Ostrowski A, Rivers CI et al (2020) Two-versus fourtimes weekly acupuncture-like transcutaneous electrical nerve stimulation for treatment of radiation-induced xerostomia: a pilot study. J Altern Complement Med 26:323-328

Publisher's Note Springer Nature remains neutral with regard to jurisdictional claims in published maps and institutional affiliations. 